\title{
THE SYSTEM OF HARVEST GETTING HIGHER USING GAME THEORY TECHNIQUE IN WIRELESS SENSOR NETWORKS
}

\section{DR. S SRIDEVI \& DR. S M CHITHRA}

Assistant Professor of Mathematics, Queens College of Arts \& Science for Women, Punalkulam, Tamil Nadu, India Associate Professor of Mathematics, RMK College of Engineering and Technology, Chennai, Tamil Nadu, India

\begin{abstract}
The game technique syndicates the augmentation and collecting, to conclude the system of government of the game theory, with fitout and to improve backbonesuitable. The method is give details classification of nourishment rate, type of nourishment and time. The game theory twisted by this system is suggestively more truthful than ordinary game theory. Additionally, a wide-ranging model adjustment study terminologies a much outranked variance for game theory than for standard game as a direct cause of the value-addedaccurateness.

KEYWORDS: Game Theory, Agricultural Data; Paddy Types
\end{abstract}

Received: Jun 08, 2020; Accepted: Jun 28, 2020; Published: Aug 28, 2020; Paper Id.: IJMPERDJUN2020921

\section{INTRODUCTION}

Every day we are faced with choices-what to dress, what to eat, what do in at liberty time performance. These choices are often made instantaneously. However, once in a blue moon a choice comes from place to place that cannot be hurriedly resolved. Possibly we cannot easily see which choice is decrease, normal or increase.

The planning of farmed networks is a relevant game theory problem that can be disintegrated in a categorization of household tasks, including the wireless sensor network design, frequency setting, development timetable growth, as well as harvest and formers setting up, in the middle of others. On the subject of crop network forecasting, a strong constraint for the harvest system is the obtainability of fertiliser. In this sense, researchers usually focus on distributing a previously defined game theory of fertiliser into a given number of orders to define a crop network.

Applications for this exact task usually consider one on its own objective function, such as the optimization of the regular time a former waits for a service. The preparation of metropolitan public agricultural systems also involves a series of real-world game theory problems that are usually tackled by human experts. One of these game theory problems is the harvest of a network in case of natural problems in fertiliser and former. For this particular task, instead of preplanning the whole harvest network if no further fertiliser or former exist, a simple solution involves studying the influence of transferring harvest or former from unaffected to affected ones.

Records show off is a collection of processes in which association among game theory is uncovered by sensor networks. The goal of data show off is to use the growth of data to recondition future hard work. Use that data as a foundation to growth model to induced future forms. One of the strong points of data show off is that it can analyze data from compound bases and give independent level growth regarding what is appropriate or not compulsory, that is for the model to agree. Model crop concern with creates a new algorithm to analysis data. The 
objective is to produce an overall instantaneous of a set of game theory data to identify and describe the main gain of features of the pattern.

\section{Game Theory Techniques Agriculture System in WSNs}

In this survey the state-of-the-art routing protocols foragriculture systemWSNs. In general, agriculture systemin WSNs can be divided into at-based routing, hierarchical-based routing, and location-based routing depending on the network structure. In at-based routing, all nodes are typically assigned equal roles or functionality. In hierarchical-based routing, however, nodes will play different roles in the agriculture system. In location-based routing, sensor nodes' positions are exploited to route data in the agriculture system.

A routing protocol is considered adaptive if certain agriculture system parameters can be controlled in order to adapt to the current network conditions and available energy levels. Furthermore, these protocols can be classified into multipath-based, query-based, negotiation-based, QoS-based, or coherent-based routing techniques depending on the protocol operation of agriculture system. In addition to the above, cooperative and non-cooperative game theory routing protocols can be classified into three categories, namely, proactive, reactive, and hybrid protocols depending on how the source ends a route to the destination.

In proactive cooperative and non-cooperative game theory protocols, all routes are computed before they are really needed, while in reactive protocols, routes are computed on demand. Hybrid protocols use a combination of these two ideas. When sensor nodes are static, it is preferable to have table driven cooperative and non-cooperative game theory routing protocols rather than using reactive agriculture system. A significant amount of energy is used in route discovery and setup of reactive protocols. Another class of routing protocols is called the cooperative and non-cooperative game theory routing protocols in agriculture system.

In cooperative and non-cooperative game theory routing, nodes send data to a central node where data can be aggregated and may be subject to further processing, hence reducing route cost in terms of agriculture system use. Many other protocols rely on timing and position information. We also shed some light on these types of protocols in this paper. In order to streamline this survey, we use a classification according to the agriculture system structure and protocol operation (routing criteria).

In the rest of this section, we present a detailed overview of the main agriculture system paradigms in WSNs. We start with network structure based protocols.

\section{Cooperative and Non-Cooperative Game Theory of Agriculture System}

The objective of energy-aware routing protocolagriculture system, a destination initiated reactive protocol, is to increase the agriculture system lifetime. Although this protocol $a_{i}$; is similar to directed diffusion, it differs in the sense that it maintains a set of crabs instead of maintaining or enforcing one optimal path at higher yields. These paths are maintained and chosen by means of a certain agriculture systemprobability.

The value of this agriculture systemprobability depends on how low the energy agriculture system of each path can be achieved. By having paths chosen at different times, the energy of any agriculture systemwill not deplete quickly. This can achieve longer agriculture system lifetime as energy is dissipated more equally among all nodes. Agriculture systemsurvivability is the main metric of this protocol. The protocol assumes that each node is addressable through a class- 
based addressing which includes the location and types of the nodes. The protocol initiates a connection through localized flooding, which is used to discover all routes between source/destination pair and their costs; thus building up the routing tables.

The high-cost yields are discarded and a forwarding table is built by choosing neighboring nodes in a manner that is proportional to their cost. Then, forwarding tables are used to send data to the destination with a probability that is inversely proportional to the node cost. Localized flooding is performed by the destination node to keep the paths alive. When compared to directed diffusion, this protocol provides an overall improvement of $25 \%$ energy saving and an $85 \%$ increase in agriculture system lifetime. However, the approach requires gathering the location information and setting up the addressing mechanism for the nodes, which complicate route setup compared to the directed diffusion.

\section{Cooperative and Non-cooperative Harvest Game}

We formulate the users' selfish behavior with a cooperative and non-cooperative game framework. Let $\mathrm{G}=$ $\left[\mathrm{N},\left(a_{\mathrm{i}}\right),\left\{\left(\mathrm{g}_{\mathrm{i}}\right)().\right\}\right]$ denote the cooperative and non-cooperative harvest game(CANHG) game where $\mathrm{N}=\{1,2, \ldots \mathrm{N}\}$ is the index set for active users currently in harvest gamenetworks, $P_{i}$ is the strategy set, and $\left\{\left(g_{i}\right)().\right\}$ is the utility function of user $a_{\mathrm{i}}$. Each users selects a power level $\mathrm{a}_{\mathrm{i}} \varepsilon A_{\mathrm{i}}$. Let the harvest game vector a $=\mathrm{a}_{1}, \mathrm{a}_{2}, \ldots \ldots \ldots \mathrm{a}_{\mathrm{N}} \varepsilon \mathrm{p}$ denote the outcome of the cooperative and Non-cooperative HarvestGame in terms of selected harvestlevels of all the users, where A is the set of all agriculture system vectors. The utility function demonstrates the strategic interdependence among users. The level of utility each user gets depends on its own harvest game level and also on the choice of other players' strategies, through the SINR of that user. We assume that each user's strategy is rational, that is, each user maximizes its own utility in a distributed fashion. Formally, the CANHGgame G is expressed as

$$
\max _{a_{i} \varepsilon A_{i}} g_{i}\left(A_{i}, A_{-i}\right) \text {, for all i\&N, }
$$

Where $\mathrm{g}_{\mathrm{i}}$ is given in (1) and $A_{i}=\left\{a_{i}^{\min } a_{i}^{\max }\right\}$ is the strategy space of user $a_{\mathrm{i}}$. In this game 'a' is the strategy profile, and the strategy profile of $i$ 's opponents is defined to be $\left(A_{-i}=A_{1}, A_{i-1}, A_{i+1}, \ldots \ldots \ldots A_{N}\right)$, so that $A=\left(A_{i}, A_{-i}\right)$.A similar notification will be used for other quantities.

Users $a_{\mathrm{i}}$ 's best response is $\mathrm{BG}_{i}\left(\mathrm{~A}_{-\mathrm{i}}\right)=\arg \max _{\mathrm{a}_{\mathrm{i}} \varepsilon \mathrm{A}_{\mathrm{i}}} \mathrm{g}_{\mathrm{i}}\left(\mathrm{A}_{\mathrm{i}}, \mathrm{A}_{-\mathrm{i}}\right)$, i.e., $A_{\mathrm{i}}$ that $\operatorname{maxg}_{\mathrm{i}}\left(\mathrm{A}_{\mathrm{i}}, A_{-\mathrm{i}}\right)$ given a fixed $A_{-\mathrm{i}}$. With the best response concept, we can present the following definition for the Nash Equilibrium (NE) of CANHG game G.

\section{Definition 1: Nash Equilibrium (NE) of CANHG Game G}

A strategy profile $p^{*}$ is a Nash Equilibrium (NE) of CANHG game G if it is a fixed point response, $\mathrm{g}_{\mathrm{i}}\left(a_{i}^{*}, a_{-i}^{*}\right) \geq$ $g_{i}\left(a_{i}^{\prime}, a_{-i}^{*}\right)$ for any $a_{i}^{\prime} \varepsilon A_{i}$ and any user $a_{\mathrm{i}}$.

The NE concept offers a predictable, stable outcome of a game where multiple agent with conflicting interests complete through self- optimization and reach a point where no player wishes to deviate. However, such a point does not necessarily exist.

\section{Definition 2: Cooperative and Non-Cooperative Harvest Game Theory}

Sensor nodes are equipped with small non-rechargeable batteries (usually less than $0.5 \mathrm{~A} \mathrm{~h}$ and $1.2 \mathrm{~V}$ ). Therefore, theefficient battery utilization of a sensor node is a critical aspect to support the extended operational lifetime of the 
individualnodes and of the whole network. A WSN routing protocol is expected to: (i) minimize the total number of communications involvedin route discovery and data delivery, and (ii) distribute the forwarding of the data packets across multiple paths, sothat all nodes can deplete their batteries at a comparable rate. This will result in the overall increase of the network lifetime.

\section{Cooperative and Non-Cooperative Harvest Game Theory}

Acooperative and Non-Cooperative Game Theory is based on the absence of coalitions in that it is assumed that each participant acts independently, without collaboration or communication without any of the others. Because cooperative and non-cooperativeharvestchoice of transmission in wireless sensor networks in the problem of cooperative and noncooperative harvestgame theory with incomplete information, we can have following result by using Bayesian Nash Equilibrium method

Theorem.3.1: Nash Equilibrium exists and is unique in cooperative and non-cooperative harvestgame with incomplete information of harvest.

\section{Proof}

Let $f_{s_{i}}(\mathrm{x})$ as the probability density function of $s_{i}$, assuming that node can carry out data transfer under any large cooperative and non-cooperative harvest, that is when $s_{i} \rightarrow \infty$, node transmission probability is 1 , so we can have $\int_{0}^{\infty} f_{s_{i}}(\mathrm{x}) \mathrm{dx}$ $=1$.

But cooperative and non-cooperative harvestgame theory in the real world, in order to reduce the payments as well as reduce cooperative and non-cooperative harvestcosts, does not allow the node forward at any big cooperative and non-cooperative harvest value. So we should let transmission cooperative and non-cooperative harvest within a certain range, we can assume that when cooperative and non-cooperative transmission harvest as $s_{i} \in\left[0, a_{i}\right]$, the $n_{i}$ will get the largest cooperative and non-cooperative networks utility. $a_{t}$ is the maximum harvest when a node transmits can be given by

$$
\mathrm{A}\left(a_{t}\right)=\int_{0}^{a_{t}} f_{s_{i}}(\mathrm{x}) \mathrm{dx}
$$

The probability of no transmission is $1-\mathrm{A}\left(a_{t}\right)$. So the probability that any k nodes out of $\mathrm{N}$ nodes are active is given by

$$
a_{n}=\sum_{k}\left(\begin{array}{l}
n \\
k
\end{array}\right)\left(\mathrm{a}\left(a_{t}\right)^{k}\right)\left(1-a\left(a_{t}\right)^{N-k}\right)
$$

Then the expected cooperative and non-cooperative networks utility of the $a_{\mathrm{i}}{ }^{t}$ node transmitting is given by

$$
\mathrm{H}\left[G_{i}^{n e t}\right]=\sum_{k=o}^{N}\left(g_{i}\left(s_{i}, s_{-i}\right)-A\left(s_{i}\right)\right) a_{k}
$$

If the node is transmitting expected cooperative and non-cooperative networks utility is equation (5). If the node dose not transmit the expected cooperative and non-cooperative networks utility is 0 . The expected cooperative and noncooperative networks utility of any node is given by

$$
\begin{aligned}
& G_{i}\left(a_{t}\right)=\int_{z_{i}}^{a_{t}}\left[G_{i}\left(a_{t}\right)-c(x)\right] f_{s_{i}}(x) d x \\
& \left.=G_{i}\left(a_{t}\right) a\left(a_{t}\right) \int_{z_{i}}^{a_{t}} c(x)\right] f_{s_{i}}(x) d x---
\end{aligned}
$$

Let $B\left(a_{t}\right)=\int_{z_{i}}^{a_{t}} c(x) f_{s_{i}}(x) d x$, then the equation (8) can be written as 


$$
\mathrm{G}_{\mathrm{i}}\left(\mathrm{a}_{\mathrm{t}}\right)=\mathrm{G}_{\mathrm{i}}\left(\mathrm{a}_{\mathrm{t}}\right) \mathrm{a}\left(\mathrm{a}_{\mathrm{t}}\right)-\mathrm{B}\left(\mathrm{a}_{\mathrm{t}}\right)
$$

From the equation (9) we can see that when the actual transmission harvest reach the value of upper bound, we get same expected utility, i.e., $s_{i}=a_{i}$. Thus $p_{i}$ is the harvest upper bound of nodes transmitting when the whole network can achieve maximum utility? That is $a_{t}$ is the solution to following equation

$$
G_{i}\left(a_{t}\right)-\mathrm{c}\left(a_{t}\right)=0
$$

\section{Simulation and Performance Analysis}

The proposed algorithm has been simulated and validated through simulation. The sensor nodes are deployed randomly in a $50 \times 50$ meters square and sink node deploy at the point of $(25,25)$, the maximum transmitting radius of each node is 40m; other simulation parameters are displayed in Table.1. In this section, we first discuss utility factor and pricing factor's influences on transmitting harvest, then evaluate the algorithm of NGLE algorithm and compare it with other existing algorithm.

Table 1: Simulation Parameters

\begin{tabular}{|l|c|l|c|}
\hline \multicolumn{1}{|c|}{ Parameters } & Value & \multicolumn{1}{c|}{ Parameters } & Value \\
\hline Transmission Range & $100 \mathrm{~m}$ & Getting Power & $18 \mathrm{~mW}(129.6 \mathrm{~J})$ \\
\hline Network Area & $50 \times 50$ & Harvest Consumption in slumber mode & $50 \mu \mathrm{W}(0.36 \mathrm{~J})$ \\
\hline Number of Sensors & $1-50$ & Transport and in receipt of Slot & $25 \mathrm{msec}$ \\
\hline Packet rate & $4 \mathrm{pkt} / \mathrm{sec}$ & Nature of mote & Mica $^{2}$ \\
\hline Packet Size & $20 \mathrm{bytes}$ & Preliminary harvest of antenna node & $1 \mathrm{KJ}$ \\
\hline Radio Bandwidth & $56 \mathrm{kbps}$ & Get-up-and-go Threshold E & $0.0001 \mathrm{~mJ}$ \\
\hline Transmitting Power & $50 \mathrm{~mW}(170 \mathrm{~J})$ & & \\
\hline
\end{tabular}

The Network Lifetime for each simulation is showed in Figure 1. These curves are showing that lifetime of network for various routing protocols after 500 rounds, about 50\% of nodes in the network are alive in the proposed REER routing protocol, but $3 \%, 10 \%$, and $15 \%$ of nodes are alive in existing protocols LHACH, LHACH-M and HEED respectively. So the network lifetime is increasing about $95 \%$ with using of our model and algorithm.

Figure 2 shows the average delivery delay with increasing harvest rate. The average delivery delay means the average time delay between the instant the source sends a packet and moment the destination receives this packet.

Table 2: Comparison LHACH Protocol and Proposed

Protocol with $E_{0}=0.25 \mathrm{~J}$

\begin{tabular}{|l|c|c|c|}
\hline \multicolumn{1}{|c|}{ Protocol } & Stability & Network & Instability \\
\hline LHACH & 440 & 700 & 250 \\
\hline Proposed protocol & 700 & 1229 & 540 \\
\hline
\end{tabular}




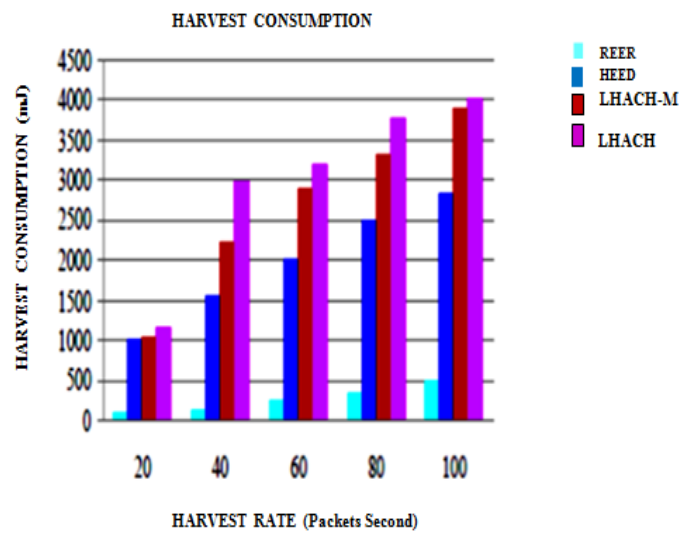

Figure 1: Harvest Expenditure with Various Consumption Rate.

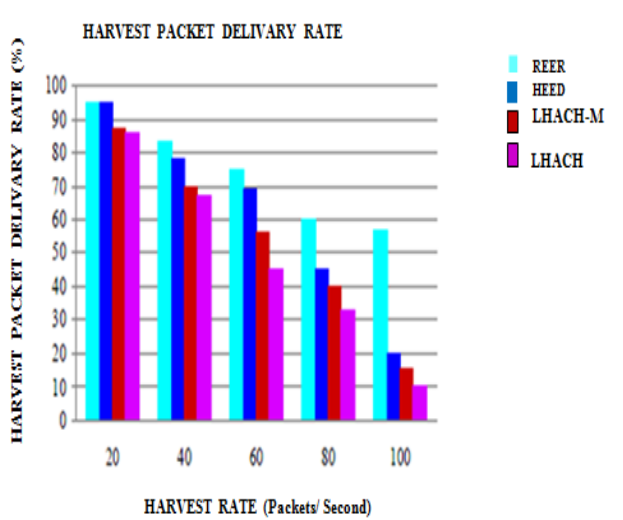

Figure 2: Harvest Packet Delivery Rate is with an Hodgepodge of Consumption Rate.

Figure.1, Figure.2, shows the harvestbalance Factor with various harvests. HarvestRate Balanced Factor (HRBF) of FAF increase slowly with light variation at first and keep stability, then increase a little time, and return to 0 as the energy of the entire harvestnetwork is using up. In this network first death of node occur at the stage of 150 rounds. HEBF is defined as the average of all nodes standard deviation based on residual energy. It's the average value of the residual energy of all of the nodes. The result of simulation show that, proposed protocol in creed network life-time up $50 \%$ compared with LHACH protocol. To evaluate the effect of the proposed protocol, we use some following parameter to measure simulation results. Those are 8\% Node Dead (TND), Haft Node Dead (HND) and Full Nodes Dead (FND).

Table 3: Compare TND, HND and FND Between LHACH and Proposed Protocol with $\boldsymbol{E}_{\mathbf{0}}=\mathbf{0 . 2 5 J}$

\begin{tabular}{|l|c|c|c|}
\hline \multicolumn{1}{|c|}{ Parameter } & 8\% Dead & 30\% Dead & 50\% Dead \\
\hline LHACH & 350 & 300 & 350 \\
\hline Proposed protocol & 400 & 400 & 610 \\
\hline
\end{tabular}

According to the simulation results, the initial harvest is $0.15 \mathrm{~J}$. In compare with LEACH protocol, proposed protocol increases TDN by $30 \%$, HND by $35 \%$ and FDN by $40 \%$.

Table 4: Compare TND, HND and FND between LHACH and Proposed Protocol with $\boldsymbol{E}_{\mathbf{0}}=\mathbf{0 . 5 J}$

\begin{tabular}{|l|c|c|c|}
\hline \multicolumn{1}{|c|}{ Parameter } & 8\% Dead & 30\% Dead & 50\% Dead \\
\hline LHACH & 400 & 556 & 640 \\
\hline Proposed protocol & 620 & 800 & 1140 \\
\hline
\end{tabular}

With initial energy $\boldsymbol{E}_{\mathbf{0}}=0.3 \mathrm{~J}$, the proposed protocol can make network lifetime increase, respectively $10 \%, 20 \%$ and $40 \%$. We see that the proposed protocols perform better than Low-HarvestAdaptive Clustering Hierarchy (LHACH) protocol.

\section{CONCLUSIONS}

In this chapter, we introduce a Cooperative and Non-Cooperative Game Theory for extending sensor network lifetime. This approach improves the harvest success rate and decreases the harvest delays of packets. In the aspect of setting up the routing path, we consider the residual harvest. We conclude the forwarding probability and payoff function of forwarding participants. We proposed a new routing protocol in order to enlarge the life-time of sensor networks. This protocol developed from LHACH protocol by considering energy and distance of nodes in WSN in CHs election. However, this 
protocol is only applied in the case of BS in the sensor area. But with BS is far from sensor area, we cannot apply this protocol. In the future, we will study the harvest distribution of node in the case BS is far from the sensor area to improve the lifetime of the whole network. Finally, the Nash Equilibrium exists when it is assumed for minimum and maximum threshold for channel condition and power level. By using Non-Cooperative and cooperative Game Theory, the harvestnetwork lifetime is extended, that is after 300 rounds, $30 \%$ of nodes are alive where as $1 \%$, $5 \%$ and $5 \%$ of nodes are alive in existing protocols LHACH, LHACH-M and HEED respectively. So the network lifetime is found to be increasing about $85 \%$ with the applications of our model and algorithm

\section{REFERENCES}

1. Jamal N. Al-Karaki Ahmed E. Kamal, "Routing Techniques in Wireless Sensor Networks: A Survey” This research was supported in part by the ICUBE initiative of Iowa State University, Ames, IA 50011, 2004.

2. I.F. Akyildiz, W. Su, Y. Sankarasubramaniam, and E. Cayirci "Wireless sensor networks: a survey”, Elsevier publications : Computer networks, vol.38,no.4,pp.393-422, 2002

3. Kemal Akkaya and Mohamed Younis, "A Survey on Routing Protocols for Wireless Sensor Networks", Ad hoc Networks, Volume 3, issue 3, pp.325-349, May 2005.

4. H.Deepa and Avan Kumar Das, “A Study on Routing Protocols in Wireless Sensor Network”, International Journal of Computer Applications, Vol.72,No.8,pp.35-39, June.2013.

5. Christian Blum and Xiaodong Li, "Swarm Intelligence in Optimization”, swarm intelligence, Springer Berlin, pp 43- 85, 2008 . 
